\title{
ICA-Derived Respiration Using an Adaptive R-peak Detector ${ }^{\star}$
}

\author{
Christina Kozia1[0000-0003-3871-5071]凶, Randa Herzallah ${ }^{10000-0001-9128-6814]}$, \\ and David Lowe ${ }^{1}$ \\ Aston University, Birmingham B4 7ET, United Kingdom \\ \{koziac,r.herzallah\}@aston. ac.uk \\ david.lowe@deeplearn.net
}

\begin{abstract}
Breathing Rate (BR) plays a key role in health deterioration monitoring. Despite that, it has been neglected due to inadequate nursing skills and insufficient equipment. ECG signal, which is always monitored in a hospital ward, is affected by respiration which makes it a highly appealing way for the BR estimation. In addition, the latter requires accurate R-peak detection, which is a continuing concern because current methods are still inaccurate and miss heart beats. This study proposes a frequency domain BR estimation method which uses a novel real-time R-peak detector based on Empirical Mode Decomposition (EMD) and a blind source ICA for separating the respiratory signal. The originality of the BR estimation method is that it takes place in the frequency domain as opposed to some of the current methods which rely on a time domain analysis, making the estimation more accurate. Moreover, our novel QRS detector uses an adaptive threshold over a sliding window and differentiates large Q-peaks from R-peaks, facilitating a more accurate $\mathrm{BR}$ estimation. The performance of our methods was tested on real data from Capnobase dataset. An average mean absolute error of less than 0.7 breath per minute was achieved using our frequency domain method, compared to 15 breaths per minute of the time domain analysis. Moreover, our modified QRS detector shows comparable results to other published methods, achieving a detection rate over $99.80 \%$.
\end{abstract}

Keywords: ICA · Frequency Domain Analysis - R-peak Detection . EMD · Local Signal Energy.

\section{Introduction}

Over the past two decades, there has been a dramatic increase in the extraction of the respiration signal and its derivative, the Breathing Rate (BR), from a single lead ECG [1]. The BR is a key indicator in health deterioration monitoring and provides valuable information on the physiological status of a person [2]. The first serious discussions and research into extraction of the respiratory

\footnotetext{
* Supported by Isansys Lifecare.
} 
signal using the ECG emerged during the 1980s and the use of the latter was suggested by reporting that a normal respiratory cycle affects the heart rate, which cause sinus arrhythmia [1]. An ECG-Derived Respiration (EDR) method was suggested in [3]. EDR uses the variations in the QRS peak amplitudes through time due to the chest movement amplitude displacements, whereas Respiratory Sinus Arrhythmia-Derived Respiration (RSA) uses the Instantaneous Heart Rate (IHR) variability which increases during inspiration and drop during exhalation due to baroreflex sensitivity [4].

More recent attention has focused on the analysis of the beat-to-beat changes in the QRS complex in order to estimate the BR. In [5] a new method for extracting the respiration signal based on the Principal Component Analysis (PCA) was suggested. It has been argued that PCA can describe the variability of the QRS complex due to respiration. Their proposed algorithm has been compared with existing EDR methods and the results were promising. In [6] an EDR method based on Independent Component Analysis (ICA) and the power spectrum of the independent components was proposed. This method is more robust as ICA assumes statistical independence between the components, whereas PCA assumes that the components are mutually uncorrelated.

As was pointed out above, it is clear that the respiration signal extraction requires an accurate QRS detector [1,3,4]. The identification of QRS complexes is a major area of interest as it is the most discernible feature of the ECG. However, R-peak detection turns out to be complex due to signal contamination from various types of noise and the morphological variability of the QRS complexes. Several studies have investigated the QRS complex detection by developing a number of methods [7-11]. The signal is pre-processed in order to amplify the QRS complex and remove noise and baseline wander and then a set of thresholds is applied in order to locate the real R-peaks in the signal. In [7] and [8] the derivative of the signal was used in order to obtain the slope and width information of the QRS complex. In [11] the Hilbert transform of the derivative of the ECG was suggested in order to locate the R-peaks. More recent studies have investigated the use of Empirical Mode Decomposition (EMD) [12] as an effective pre-processor which amplifies the QRS complex and decomposes the signal into a set of Intrinsic Mode Functions (IMF) [13-15]. A serious weakness of the above methods is that the threshold is derived from the full length ECG. Generally difficulties arise when the signal includes very large R-peaks, making the threshold high. This results in the failure to detect lower R-peaks.

The current study proposes a novel QRS detector which overcomes the aforementioned problems in the current state-of-the-art EMD methods by introducing an adaptive threshold which is calculated from the local energy of the reconstructed ECG signal from the EMD. The pre-processing stage contains a band-pass filter which eliminates noise and reduces the number of the IMFs. The reconstruction of the signal using the EMD method facilitates the removal of low frequency interference and the absolute value of the reconstructed signal amplifies the QRS complexes. The signal is then divided into segments in order to increase the efficiency of the algorithm. Compared with the existing results 
on the topic, our study has three distinct features that have not been reported in the literature. Firstly, the proposed detector provides a solution for the detection of small R-peaks by establishing a threshold derived from the mean of the root mean square (rms) over a pre-specified number of most recent segments. Secondly, the threshold established relies on the local signal energy of each segment. Thirdly, the present research explores the differentiation of R-peaks from large Q-peaks in the absolute value of the signal, by using the first derivative of the ECG signal.

Based on the ECG respiratory-induced modulations a statistical signal processing approach will be considered for this study: the ICA analysis of the beatto-beat variations during respiration. The method requires the locations of the R-peaks identified by our QRS detector, which are stacked into a matrix for further analysis. Then ICA is applied and the Independent Component (IC) which corresponds to respiration is identified based on its frequency spectrum. The respiratory IC is then filtered in order to derive the ICA respiration signal. The latter is then divided into one minute windows in order to estimate the BR. For each window the Fourier transform of the respiration signal is computed. The novelty of this work lies on the fact that the BR estimation takes place in the frequency domain, whereas time domain analysis is sensitive to double-humped peaks, which makes BR estimation inaccurate. Moreover, our frequency domain approach can be implemented in real-time as the estimation requires windows of one minute duration. Finally, the identification of the respiratory IC lies on the analysis of the respiratory frequencies, making the BR estimation more accurate.

\section{Proposed QRS Detector}

The main assumption of our proposed R-peak detector is that the QRS complex can be further amplified by reconstructing the signal from its first three IMFs after applying the EMD algorithm. Our assumption has been verified on all the tested recordings as well be shown in Section 2.2.

\subsection{Empirical Mode Decomposition}

EMD decomposes the signal, $x(t)$, into a series of narrow-band signals, $c_{i}(t)$, which are called IMFs, and fulfil special conditions. An oscillatory mode of the signal is an IMF exclusively under the conditions that: first, in the whole dataset, the number of zero-crossings and the number of extrema are either equal of differ at most by one; and second, at any point, the mean value of the maximum and the minimum envelope is zero. The key advantage of EMD is that it is a data-driven analysis method. At each iteration the algorithm needs to decide if the $i$-th component, $h_{i}(t)$, extracted from the data, is an IMF based on the conditions mentioned above. In order to achieve this, the EMD method uses a systematic way which is called the sifting process and is described as follows: for a given signal $x(t)$, the extrema points are first identified, followed by approximation of the upper, $\hat{r}(t)$, and lower, $\underline{r}(t)$, envelopes of the signal through a cubic spline interpolation. The mean of the envelopes is then obtained, and 
the $i$-th component, $h_{i}(t)$, is computed as the difference between the signal and the mean. The sifting process has to be repeated as many times as required to reduce the extracted signal to an IMF. For our implementation, in order to stop the sifting process, the number of zero-crossings and the number of extrema are checked for equality or whether they differ at most by one. If the final residue, $r_{N}(t)$, is obtained as a monotonic function, the EMD algorithm is terminated, $c_{i}=h_{i}$, and the signal, $x(t)$, can be written as follows:

$$
x(t)=\sum_{i=1}^{N} c_{i}(t)+r_{N}(t),
$$

where $N$ is the total number of the extracted IMFs.

\subsection{QRS Detector Algorithm}

To summarise, the proposed QRS detector is as follows:

\section{Pre-processing Stage}

1. The raw signal, $x(t)$, is first filtered with a band-pass filter, whose coefficients were designed using a Kaiser-Bessel window [16]. The band-stop frequencies were set at 8 and $20 \mathrm{Hertz}$ [17] in order to amplify the QRS complex, eliminate noise and reduce the number of IMFs. The output of the filter is denoted as $x_{f}(t)$,

2. The EMD algorithm is applied on $x_{f}(t)$ to extract the IMFs, $c_{1}(t), \ldots, c_{N}(t)$, where $N$ is the total number of the extracted IMFs,

3. The signal is reconstructed by adding the first three IMFs,

$$
x_{r}(t)=\sum_{i=1}^{3} c_{i}(t),
$$

where the number of IMFs is experimentally selected and it will be discussed later,

4. Then, the absolute value of the reconstructed signal is computed, that is $a(t)=\left|x_{r}(t)\right|$. This makes all data points positive and implements a linear amplification of the reconstructed signal emphasising the higher cardiac frequencies, such as the QRS complex.

\section{Decision Stage}

5. In order to increase the efficiency of the algorithm, we divide $a(t)$ into $k$ segments of 3 seconds duration, that is $k=$ (total number of samples) $/(3 *$ $f s)$. The starting point of the $k$-th segment should match the last R-peak located on the $k-1$ segment in order to increase the accuracy,

6. Compute the envelope of the maxima, $\hat{a}_{k}(t)$, of $a_{k}(t)$ for each segment $k$ through a cubic spline interpolation of the local maxima, 
7. Compute the local signal energy for each segment as,

$$
R M S_{k}=\sqrt{\frac{1}{M} \sum_{t=1}^{M}\left[\hat{a}_{k}(t)\right]^{2}},
$$

where $k$ is the current segment and $M$ is the number of samples in the segment, that is $M=3 * f s$,

8. The threshold of the $k$-th segment is set to be the mean of the eight most recent $R M S_{k}$ values,

$$
T_{k}=\frac{1}{8} \sum_{j=k-7}^{k} R M S_{j},
$$

9. The peaks, which exceed threshold $T_{k}$ in the absolute sequence $a_{k}(t)$, are classified as candidate peaks,

10. In order to segregate large Q-peaks from R-peaks, we compute the first derivative of $x_{r}(t)$. Peaks with a negative derivative will be investigated further at the refractory period check given next,

11. Apply a refractory period check when the R-R interval of two adjacent peaks is less than 200 milliseconds. Keep the peak with the maximum amplitude.

The main assumption of the proposed R-peak detector is that the first three IMFs can be used in the QRS complex amplification, because their frequency spectra correspond to the QRS frequencies (3-40 Hz [18]). Furthermore, the IMFs which represent slower oscillations, like P- or T- waves $(0.7-10 \mathrm{~Hz}$ [18]), are omitted in order to enhance the R-peaks. To enhance the QRS complex and reduce computation and the number of the extracted IMFs, it is recommended that the ECG signal is filtered with a band-pass filter. Fig. 1 shows a filtered ECG signal and its first five IMFs, obtained after the EMD algorithm. It is evident from Plots 7 to 11 that as the order of the IMFs increases, the frequency of the mode decreases. It is also shown that the spectra of the first three IMFs correspond to the frequency band of the QRS complex. Fig. 2 shows that the filtered signal, $x_{f}(t)$, can be approximated by the reconstructed signal, $x_{r}(t)$, because the difference of the two signals (yellow dotted line) is small and the shape of the QRS complex is preserved.

Our study used a convenience sample of 20 children recordings (median age: 9.1, range 1-16 years) and 5 adult recordings (median age: 46.2, range 37-64) from the Capnobase dataset [19]. The latter contains ECG, pulse oximetry and capnoraphy recordings of 8 minutes duration acquired during elective surgery and routine anaesthesia. The sampling frequency, which was used for the ECG acquisition, was set to be at $300 \mathrm{~Hz}$. Furthermore, the dataset contains fully annotated data, providing the actual R-peaks and reference BR data. The results obtained from our proposed QRS detector are shown in Table 1. Table 2 shows a comparison of our method's performance with other existing detectors. Fig. 2 shows the sequential steps of the R-peak detection method. The detected Rpeaks are marked by an asterisk '*' on the filtered signal, $x_{f}(t)$ (Plot 4$)$. A false negative (FN) occurs when the algorithm fails to detect an actual R-peak. A false positive (FP) represents a false peak detection. Sensitivity (Se), Positive 

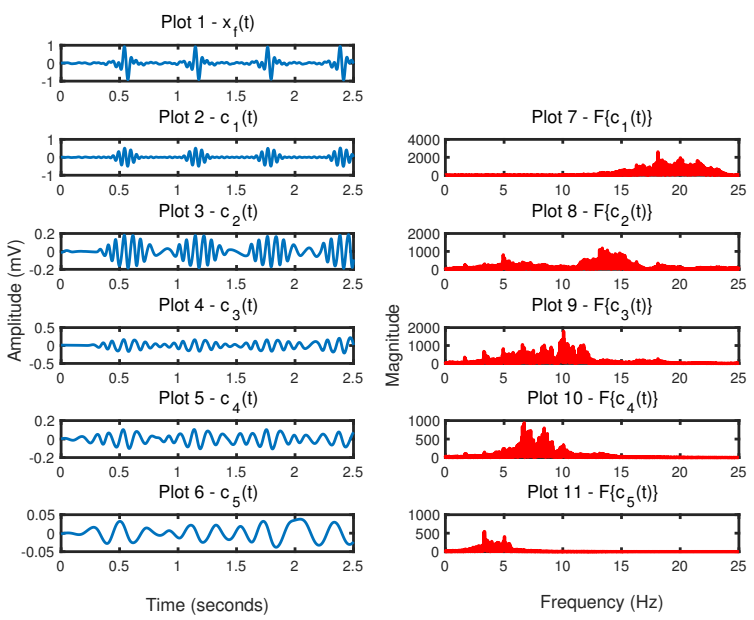

Fig. 1: The result on the EMD and the spectrum of each IMF. Plot 1 corresponds to the filtered ECG, $x_{f}(t)$. Plots 2 to 6 correspond to the first five IMFs. Plot 7 to 11 correspond to the Fourier transform of each IMF.

Predictivity $(+\mathrm{P})$ and Detection Error Rate (DER) were calculated for each recording using the following formulas respectively:

$$
\begin{gathered}
S e(\%)=\frac{T P}{T P+F N} \%, \\
+P(\%)=\frac{T P}{T P+F P} \%, \\
D E R(\%)=\frac{F P+F N}{\text { total number of R peaks }} \%,
\end{gathered}
$$

where TP (true positives) is the total number of R-peaks correctly identified. As can be seen from Table 1 and Table 2 our QRS detector shows a better performance for the Capnobase records, achieving a Se of $100 \%$, a higher $+P$ of $99.80 \%$ compared to $99.78 \%$ in [7] and $99.70 \%$ in [11], as well as a lower DER of $0.24 \%$ compared to $0.25 \%$ in [7] and $0.31 \%$ in [11].

An important observation is that the absolute amplitude of a Q-peak occurs to be larger than the R-peak in some cases. This was found to identify the Q-peak as a real R-peak, because the threshold is applied to the absolute waveform. Therefore, the first derivative of the ECG signal is computed which facilitates the Q-, R-peak segregation. Peaks with a negative derivative will be investigated further in the decision stage by applying the refractory period check. Secondly, another significant advantage of our proposed method is that it can be implemented in real-time with a detection delay equal to the duration of the segment. On-line implementation requires a small alteration of our method. The segmentation can be executed at the very beginning of the pre-processing stage and the sequential steps of our algorithm can be implemented for each segment. 

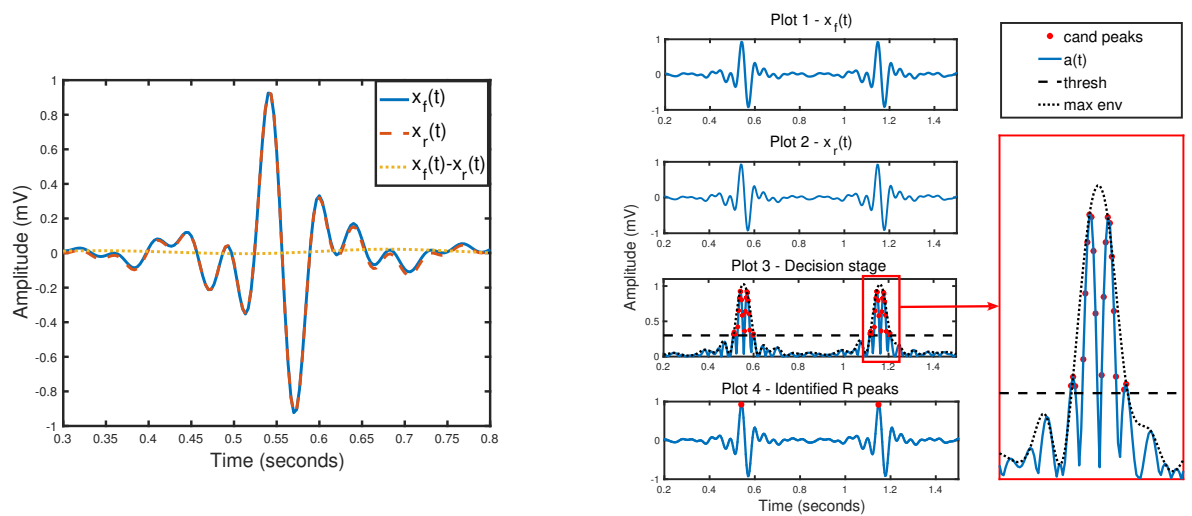

Fig. 2: Left plot - Reconstruction of the filtered signal, $x_{f}(t)$, by the summation of the first three IMFs, $x_{r}(t)$, and their difference, $x_{f}(t)-x_{r}(t)$. Right plot - Steps of the QRS detector. Plot 1, corresponds to the filtered ECG signal, $x_{f}(t)$. Plot 2, corresponds to the reconstructed signal, $x_{r}(t)$. Plot 3 , shows the absolute sequence, $a_{k}(t)$, (blue line) and its maximum envelope, $\hat{a}_{k}(t)$, (dotted black line) along with the threshold (dashed black horizontal line) and candidate peaks marked with a red asterisk '*'. Plot 4 , shows the identified $\mathrm{R}$ peaks on $x_{f}(t)$ as red asterisk ' $*$ '.

Table 1: QRS detection performance using the Capnobase Dataset

\begin{tabular}{|lllll|}
\hline Rec & Annotated peaks & DER $(\%)$ & $\boldsymbol{S e}(\%)$ & $+\boldsymbol{P}(\%)$ \\
\hline 9 & 815 & 0.00 & 100 & 100 \\
15 & 960 & 0.00 & 100 & 100 \\
16 & 1012 & 0.00 & 100 & 100 \\
18 & 1131 & 0.00 & 100 & 100 \\
23 & 818 & 0.00 & 100 & 100 \\
32 & 685 & 0.00 & 100 & 100 \\
35 & 900 & 0.18 & 100 & 99.89 \\
38 & 956 & 0.00 & 100 & 100 \\
103 & 826 & 0.00 & 100 & 100 \\
104 & 912 & 0.00 & 100 & 100 \\
105 & 530 & 0.37 & 100 & 99.62 \\
121 & 579 & 0.00 & 100 & 100 \\
122 & 588 & 0.00 & 100 & 100 \\
125 & 627 & 0.00 & 100 & 100 \\
127 & 615 & 0.00 & 100 & 100 \\
128 & 541 & 0.18 & 100 & 99.82 \\
134 & 578 & 1.53 & 100 & 98.50 \\
142 & 739 & 0.00 & 100 & 100 \\
147 & 538 & 3.58 & 100 & 97.52 \\
148 & 624 & 0.00 & 100 & 100 \\
311 & 555 & 0.17 & 100 & 99.82 \\
312 & 432 & 0.00 & 100 & 100 \\
313 & 588 & 0.00 & 100 & 100 \\
322 & 589 & 0.16 & 100 & 99.83 \\
325 & 584 & 0.00 & 100 & 100 \\
\hline \hline Average & $\mathbf{1 7 7 1 6}$ & $\mathbf{0 . 2 4}$ & $\mathbf{1 0 0}$ & $\mathbf{9 9 . 8 0}$ \\
\hline & & & & \\
\hline
\end{tabular}


Table 2: Comparison of QRS detector performance with other methods for Capnobase Dataset

\begin{tabular}{|cccc|}
\hline Method & DER (\%) & $\boldsymbol{S e}(\%)$ & $\boldsymbol{+ P}(\%)$ \\
\hline Derivative based [7] & 0.25 & 100 & 99.78 \\
Hilbert transform [11] & 0.31 & 100 & 99.70 \\
Our method & $\mathbf{0 . 2 4}$ & $\mathbf{1 0 0}$ & $\mathbf{9 9 . 8 0}$ \\
\hline
\end{tabular}

\section{Proposed BR Estimation}

The main idea of our proposed BR estimation method is to analyse the beat-tobeat changes in the QRS complex in order to extract the respiration signal using the ICA. In order to derive the respiration signal, the R-peaks are located using our proposed QRS detector (Section 2). The QRS complexes are then stacked into a matrix and then the ICA is applied. The novelty of this method is that the identification of the IC which corresponds to respiration takes place in the frequency domain. The respiration signal is then filtered in order to derive the final ICA respiration signal. The importance and originality of our proposed BR estimation method are that it explores the respiration signal in the frequency domain, whereas existing BR estimation methods are focused on a time domain analysis which can cause inaccuracies [20]. The main assumption of our method is that the most dominant peak of the respiration frequency spectrum corresponds to the BR.

\subsection{Independent Component Analysis}

Blind Source Separation (BSS) represents a model where a set of unobserved source signals is been recovered from a set of observed mixtures $[21,22]$. In other words, BSS is the separation of a set of source signals, $s(t)=\left\{s_{1}(t), s_{2}(t), \cdots, s_{n}(t)\right\}$, from a set of mixed signals, $x(t)=$ $\left\{x_{1}(t), x_{2}(t), \cdots, x_{m}(t)\right\}$. In ECG signal processing, we assume that the sources are signals like the electrical heart activity, the respiration and some noise, which are mixed, and give as result the observed ECG (Fig.3). Since it was reported in 1991, ICA has been attracting a lot of interest in machine learning and signal processing [23]. ICA is a statistical approach whose main assumption is that the observations are actually linear combinations of statistically independent variables $[21,24]$, as follows:

$$
x_{i}=a_{i, 1} s_{1}+\cdots+a_{i, k} s_{k}+\cdots+a_{i, n} s_{n},
$$

where $\boldsymbol{a}_{k}$ are the basis vectors which form the columns of the mixing matrix $\boldsymbol{A}$ (Fig. 3 ) and $i=1, \cdots, m$.

\subsection{ICADR Algorithm}

To summarise, the proposed ICA-Derived Respiration (ICADR) method is as follows: 


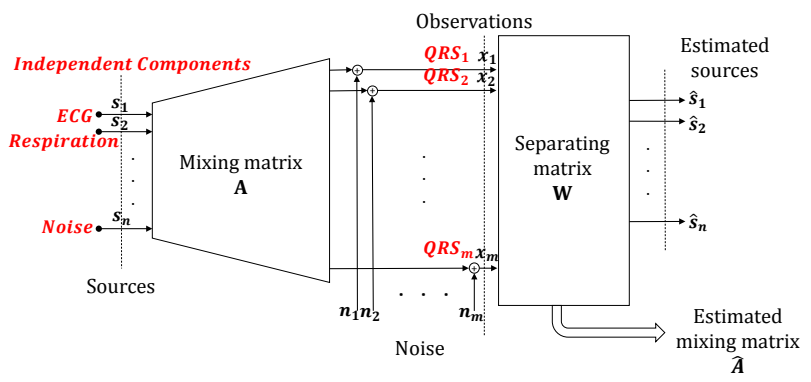

Fig. 3: Schematic representation of the BSS model in ECG signal processing

1. Extract the R-peaks from a single-lead ECG signal using our proposed QRS detector,

2. Create the data matrix and whiten the data,

3. Apply PCA method for dimensionality reduction and keep the first 10 Principal Components (PC),

4. Apply the fastICA algorithm to the reduced matrix in order to get the source signals as independent components (Fig. 4),

5. Compute the Fourier transform of the ICs produced and choose as respiration signal the IC whose frequencies lie between reasonable respiration rates (Fig. 4),

6 . Filter the output of step 5 within reasonable respiration frequencies $(0.0666$ $0.5 \mathrm{Hertz}$ ) to get the ICA respiration signal,

7. Divide the respiration signal into one minute windows,

8. For each window compute the Fourier transform and locate the frequency that corresponds to the most dominant peak in the frequency spectrum (Fig. $5)$,

9. Convert frequency to bpm to get the BR.

The data matrix construction is based on the fact that we are interested in analysing the inter-beat variations of the ECG features. Using the detected R-peaks (Section 2.2) the data matrix $\boldsymbol{X}$ is constructed from a singlelead ECG by stacking $m$ segments of length $n$ around the R-peak, that is, $\boldsymbol{X}_{m \times n}=\left[\boldsymbol{x}_{1} \ldots \boldsymbol{x}_{m}\right]^{T}$, where $\boldsymbol{x}_{i}$ is a row vector of length $n$, that is $\boldsymbol{x}_{i}=$ $\left[x_{i, 1} \cdots x_{i, j} \cdots x_{i, n}\right]$. Hence, the first row contains $n$ samples around the first R-peak, the second row contains $n$ samples around the second R-peak etc. For recording 0009 , the detected $\mathrm{R}$-peaks are 815 . We are taking 100 points around the R-peak, thus the size of the matrix is $815 \times 100$. After applying the PCA for dimensionality reduction the size of the matrix reduces to $10 \times 815$.

For all the recordings the results obtained from our ICADR method are shown in Table 3. In order to evaluate the performance of our method, we computed 

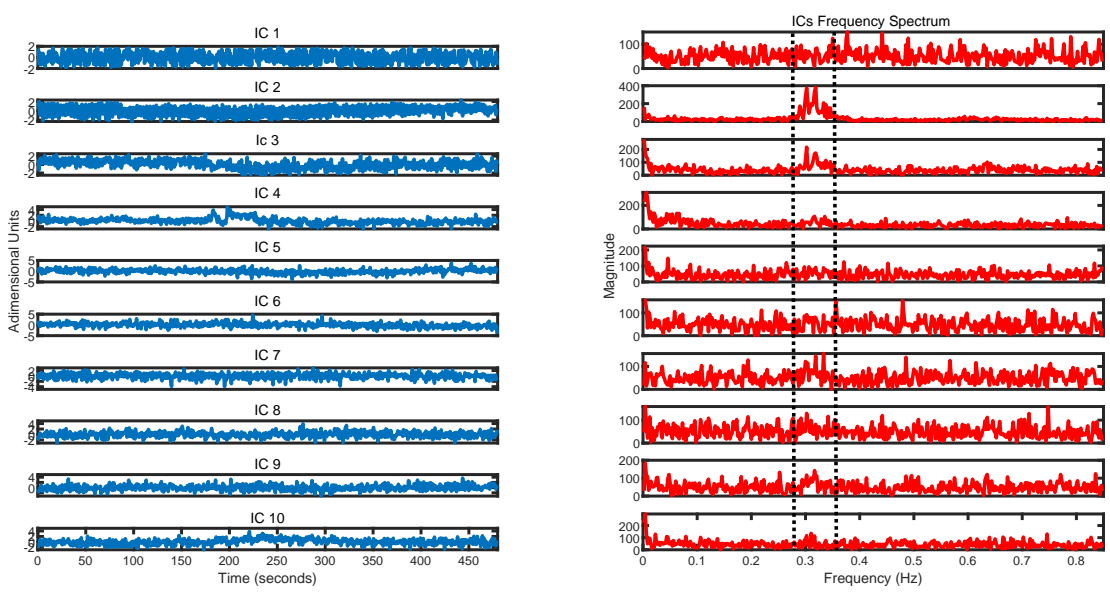

Fig. 4: The plots on the left show the 10 extracted ICs (blue) from an ECG recording and the plots on the right show the corresponding frequency spectra (red). The vertical dotted lines define the frequency range of the IC2, which is chosen to be the ICA respiration signal, because its frequencies lie between reasonable respiration rates (12$24 \mathrm{bpm})$.

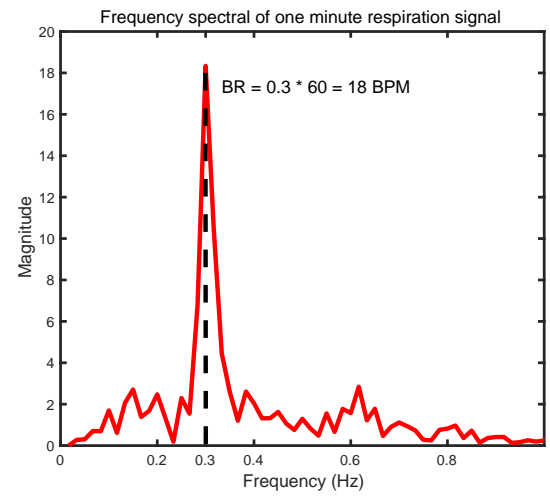

Fig. 5: The frequency spectrum of one minute window respiration signal. The dominant peak is located (black dashed line) and it is converted to breaths per minute (bpm). 
the Mean Absolute Error (MAE) in bpm, as follows:

$$
M A E=\frac{1}{N} \sum_{i=1}^{N}\left|B R_{\text {ref }_{i}}-B R_{e s t_{i}}\right|,
$$

where $N$ is the number of the extracted BRs, $B R_{\text {ref }}$ and $B R_{\text {est }}$ are the reference and estimated BR respectively. The obtained ICA respiration signal was analysed using our proposed frequency domain analysis for the BR estimation. Furthermore, the respiration signal was later upsampled at $8 \mathrm{~Hz}$ and then filtered using a band-pass filter within a reasonable respiration frequency range. It is evident from our results that the post-processing stage improved the accuracy of the estimation about $0.2747 \mathrm{bpm}$, achieving an average MAE of $0.6551 \mathrm{bpm}$. Fig. 6 shows the respiration signal obtained from our method for recording 0009, along with the frequency domain analysis, which shows a clear dominant peak in each window. It can be also observed that the error for recording 0147 is high about $8 \mathrm{bpm}$, and after the post-processing stage it drops to $5 \mathrm{bpm}$. One possible explanation is that the existence of false positives in the R-peak detection affects the accuracy. For recording 0147 the number of false positives was 13 compared to 0 false positives for recording 0009 .

Table 3: ICA-Derived Respiration Evaluation Performance

\begin{tabular}{|c|c|c|c|}
\hline & \multicolumn{2}{|c|}{ Frequency Domain } & Time Domain \\
\hline & No post-processing & Post-processing & \\
\hline Children Recordings & \multicolumn{2}{|c|}{ MAE (bpm) } & MAE (bpm) \\
\hline 0009 & 0.6359 & 0.5109 & 7.2088 \\
0015 & 0.2086 & 0.2086 & 1.0354 \\
0016 & 0.0048 & 0.0048 & 37.4550 \\
0018 & 1.2587 & 1.3837 & 14.8083 \\
0023 & 0.4867 & 0.6117 & 8.9675 \\
0032 & 2.3126 & 1.2261 & 18.1932 \\
0035 & 2.1316 & 1.9887 & 17.0079 \\
0038 & 1.6166 & 1.6166 & 16.8503 \\
0103 & 0.0079 & 0.0079 & 22.3282 \\
0104 & 0.0049 & 0.0049 & 22.0298 \\
0105 & 0.0276 & 0.0276 & 12.8290 \\
0121 & 0.0069 & 0.0069 & 13.2939 \\
0122 & 0.0070 & 0.0070 & 19.5208 \\
0125 & 0.3816 & 0.3623 & 15.0810 \\
0127 & 0.4262 & 0.4262 & 7.5363 \\
0128 & 0.3477 & 0.3477 & 11.7071 \\
0134 & 0.3788 & 0.5038 & 13.4641 \\
0142 & 0.1368 & 0.1368 & 18.4440 \\
0147 & 8.8772 & 5.3786 & 19.2597 \\
0148 & 0.0051 & 0.0051 & 19.5607 \\
\hline \hline 0311 & & & \\
\hline 0312 & 1.8822 & 0.8878 & 14.4469 \\
0322 & 0.0096 & 0.0096 & 12.3631 \\
\hline Average & 0.0110 & 0.0110 & 8.8237 \\
& 0.5638 & 0.5648 & 4.2980 \\
\hline Adult Recordings & 1.5150 & $\mathbf{0 . 6 5 5 1}$ & $\mathbf{1 5 . 0 0 0 0}$ \\
\hline
\end{tabular}



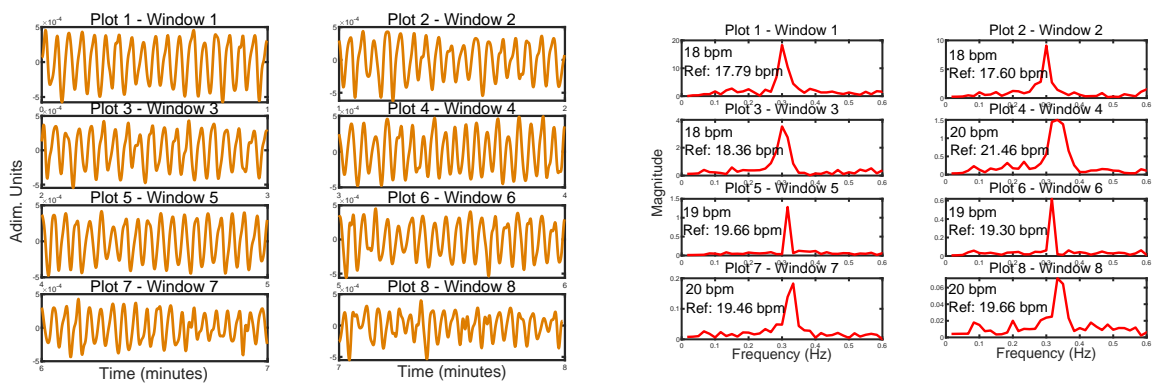

Fig. 6: Left plot - The respiration signal obtained from our ICADR method for recording 0009. Plot 1 to 8 correspond to the 8 one minute windows obtained from our algorithm. Right plot - Frequency domain analysis of the respiration signal for recording 0009. Plot 1 to 8 show the frequency spectra of each one minute window, along with the reference BR.

In order to prove that our BR estimation method in the frequency domain outperforms the time domain analysis [20], we compared our approach with the time domain of the respiration signal. The results obtained are shown in Table 3. A basic peak detector is used in order to identify the peaks in the respiration signal, along with their time locations [20]. The evaluation of the Instantaneous Respiration Rate (IRR) from the time intervals of consecutive peaks in the respiration signal is attempted. As soon as the IRRs are computed, they are converted to bpm in order to estimate the BR. It can be observed that the MAE obtained from the time domain analysis is high about $15 \mathrm{bpm}$. The major drawback of this method is that the existence of double-humped peaks in the respiration signal affects the peak detection.

\section{Conclusion}

To conclude, a new ICA-Derived Respiration (ICADR) method was presented based on an accurate and adaptive R-peak detector. Moreover, a frequency domain analysis of the respiration signal was proposed in this study for the BR estimation. The ICADR achieved a low average MAE of $0.6551 \mathrm{bpm}$ for both children and adult recordings. The frequency domain analysis outperforms the $\mathrm{BR}$ estimation in the time domain. The latter method gave a high average MAE of $15 \mathrm{bpm}$. This emphasises the importance and novelty of the proposed frequency domain analysis method.

The present study also suggested a new R-peak detector based on the EMD of the ECG signal and an adaptive threshold which relies on the local signal energy. Our method facilitates the detection of small R-peaks by deriving a threshold from the mean of the rms over eight segments. The decision stage also contains gradient-based and refractory period checks to differentiate large Q-peaks and omit false R-peaks. For Capnobase dataset, the R-peak detector 
performed effectively with accurate detection of $99.80 \%$, showing a comparable performance with other existing methods.

\section{References}

1. Moody, G.B., Mark, R.G., Zoccola, A., Mantero, S.: Derivation of respiratory signals from multi-lead ECGs. Computers in cardiology, 12(1985), 113-116 (1985)

2. Goldhill, D.R., McNarry, A.F., Mandersloot, G., McGinley, A.: A physiologicallybased early warning score for ward patients: the association between score and outcome. Anaesthesia, 60(6), 547-553 (2005)

3. Babaeizadeh, S., Zhou, S.H., Pittman, S.D., White, D.P.: Electrocardiogram-derived respiration in screening of sleep-disordered breathing. Journal of electrocardiology, 44(6), 700-706 (2011)

4. Helfenbein, E., Firoozabadi, R., Chien, S., Carlson, E., Babaeizadeh, S.: Development of three methods for extracting respiration from the surface ECG: a review. Journal of electrocardiology, 47(6), 819-825 (2014)

5. Langley, P., Bowers, E.J., Murray, A.: Principal component analysis as a tool for analyzing beat-to-beat changes in ECG features: application to ECG-derived respiration. IEEE Transactions on Biomedical Engineering, 57(4), 821-829 (2010)

6. Tiinanen, S., Noponen, K., Tulppo, M., Kiviniemi, A., Seppänen, T.: ECG-derived respiration methods: Adapted ICA and PCA. Medical engineering \& physics, 37(5), 512-517 (2015)

7. Pan, J., Tompkins, W.J.: A real-time QRS detection algorithm. IEEE Transactions on Biomedical Engineering, 32(3), 230-236 (1985)

8. Hamilton, P.S., Tompkins, W.J.: Quantitative investigation of QRS detection rules using the MIT/BIH arrhythmia database. IEEE Transactions on Biomedical Engineering, 12, 1157-1165 (1986)

9. Maglaveras, N., Stamkopoulos, T., Diamantaras, K., Pappas, C., Strintzis, M.: ECG pattern recognition and classification using non-linear transformations and neural networks: A review. International Journal of Medical Informatics, 52(1-3), 191-208 (1998)

10. Kohler, B.U., Hennig, C., Orglmeister, R.: The principles of software QRS detection. IEEE Engineering in Medicine and Biology Magazine, 21(1), $42-57$ (2002)

11. Benitez, D.S., Gaydecki, P.A., Zaidi, A., Fitzpatrick, A.P.: A new QRS detection algorithm based on the Hilbert transform. Computers in Cardiology, 27, 379-382 (2000)

12. Huang, N.E., Shen, Z., Long, S.R., Wu, M.C., Shih, H.H., Zheng, Q., Yen, N.C., Tung, C.C., Liu, H.H.: The Empirical Mode Decomposition and the Hilbert spectrum for nonlinear and non-stationary time series analysis. In Proceedings of the Royal Society of London A: Mathematical, Physical and Engineering Sciences, 454(1971), 903-995 (1998)

13. Yang, X.L., Tang, J.T.: Hilbert-Huang transform and Wavelet transform for ECG detection. In: 4th International Conference on Wireless Communications, Networking and Mobile Computing, pp. 1-4, IEEE, Dalian, China (2008)

14. Arafat, M.A., Hasan, M.K.: Automatic detection of ECG wave boundaries using Empirical Mode Decomposition. In: 34th International Conference on Acoustics, Speech and Signal Processing, pp. 461-464, IEEE, Taipei, Taiwan (2009)

15. Taouli, S.A., Bereksi-Reguig, F.: Detection of QRS complexes in ECG signals based on Empirical Mode Decomposition. Global Journal of Computer Science and Technology, 11(20), (2011) 
16. Proakis, J.G. and Manolakis, D.G.: Digital signal processing: Principles, Algorithms, and Applications 3rd edition. Prentice Hall (1996)

17. Elgendi, M., Jonkman, M. and DeBoer, F.: Frequency bands effects on QRS detection. 3rd International Joint Conference on Biomedical Engineering Systems and Technologies, 5, pp. 428-431 (2010)

18. Malmivuo, J., Plonsey, R.: Bioelectromagnetism: principles and applications of bioelectric and biomagnetic fields. Oxford University Press, USA (1995)

19. Karlen, W., Raman, S., Ansermino, J.M. and Dumont, G.A.: Multiparameter respiratory rate estimation from the photoplethysmogram. IEEE Transactions on Biomedical Engineering, 60(7), pp. 1946-1953 (2013)

20. Shah, S.A.: Vital sign monitoring and data fusion for paediatric triage, Doctoral dissertation, University of Oxford, 2012

21. Jutten, C., Herault, J.: Blind separation of sources, part I: An adaptive algorithm based on neuromimetic architecture. Signal processing, 24(1), 1-10 (1991)

22. Cardoso, J.F.: Blind signal separation: statistical principles. Proceedings of the IEEE, 86(10), 2009-2025 (1998)

23. Tanskanen, J.M., Viik, J.J.: Independent Component Analysis in ECG Signal Processing. In Advances in Electrocardiograms-Methods and Analysis, InTech (2012)

24. Comon, P.: Independent component analysis, a new concept?. Signal processing, 36(3), 287-314 (1994) 